\title{
Image Analysis using LU, SVD and Kronecker Algebra in Multimodal Biometric Authentication System
}

\author{
Y Suresh \\ Department of Information Technology ${ }^{1,2}$, PVP Siddhartha Institute of Technology, Vijayawada, India. \\ Email: sureshyadlapati@gmail.com
}

\begin{abstract}
Accurate uniqueness validation is critical in several aspects of our life. In early days prior to computing era, security is ensured by verifying the person personally and also with the help of the signature. Traditional authentication is inefficient as anyone can imprint as a real person with medical procedure and through spoofing. Now a day's authentication is done by online or offline by capturing the unique features such as biometrics of a person. The advantage of these unique in nature so that no one can represent the real person. To process these biometric qualities to achieve authentication is very complex. To improve the exactness, researches proposed different algorithms.

In this paper, by considering face and finger of a person and by using decomposing and reconstruction techniques such as Lower and Upper (LU) factorization, Singular Value Decomposition (SVD), and applications of Kronecker product we implemented multimodal authentication system. This system gives better result when compared to others.
\end{abstract}

Index Terms- Biometric, LU factorization, SVD, Katri Rao product and Kronecker product.

\section{INTRODUCTION}

Our society is electronically connected with the rapid growth of Information technology. With the vast usage of inter-connected electronic devices the daily transactions happened between individuals and organization such as banking and so on is increasing at stupendous rate. Biometric identification is gaining more importance day by day. Now, biometric indicators such as hand geometry, hand vein, face, facial thermo gram, fingerprint, ear, iris, signature, and voice print are widely used by the researchers for intensive evaluation [1]. Different mathematical models are available and each of them having its own advantages and disadvantages related to the acceptance and performance [2].

Authentication systems using biometrics can be categorized in two ways: One is unimodal biometric system - in this single biometric trait can be used for authentication and another is multimodal authentication system by using combination of two or more biometric traits. In order to operate biometric system efficiently for authentication in diverse environments, a multimodal biometric system [3] is preferred.

The proposed multimodal biometric authentication system works by pre-processing the image using Principle Component Analysis (PCA) and then preprocessed image patterns will be represented as vector using vector algebra for the feature extraction. Feature extraction is completed using Lower and Upper (LU) factorization and Singular Value Decomposition (SVD). Finally, we applied convolution technique Katri Rao product which is a variant of Kronecker product for evaluating the features. An overview of the computational framework for the proposed model is described in figure 1.

\section{FEATURE SELECTION AND EXTRACTION}

\subsection{Principal Component Analysis}

Principal component analysis is a algebraic method for interpretation in multiple channels through an

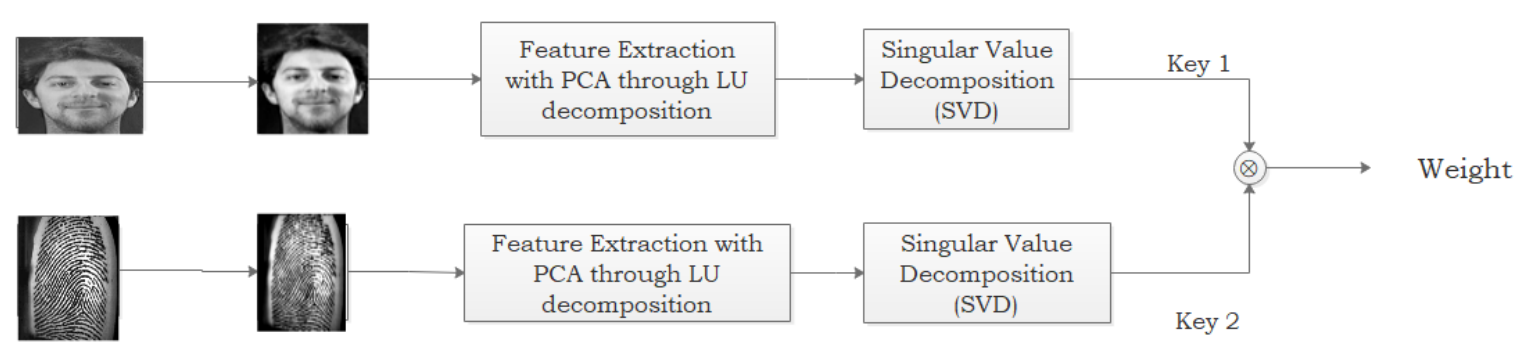

Fig. 1 Framework of the proposed model

orthonormal projection. Not only does principal component analysis have the ability to remove 


\section{Available online at www.ijrat.org}

uncorrelated noise, but also to decompose an image into constituent components. PCA has been in different applications including data transmission, compression and digital image enhancement [6]. PCA destructs a multi channel signal or an image into a set of orthogonal bases of decreasing energy. These sets can be reconstructed into the original signal exactly, or a truncated set can be used in reconstruction that tends to eliminate noise.

If the matrix of eigen vectors sorted according to eigen value by $\widetilde{U}$, then PCA transformation of the data as $\mathrm{Y}=\widetilde{\mathrm{U}}^{\mathrm{T}} \mathrm{X}$. The eigen vectors are called principle components [5].

\subsection{LU Factorization}

Lower and Upper (LU) decomposition of a matrix representation is a mathematical method in the field of Image Processing and signal processing[4]. It is the multiplication of lower and upper triangle matrices. Sometimes it can also be considered as Gaussian Elimination.

Let $\mathrm{A}$ be a NxN matrix, it can be written as a product of lower( L) and upper (U) matrices as $\mathrm{LU}=\mathrm{A}$

ie

$\left[\begin{array}{ccc}\mathrm{l}_{11} & 0 & 0 \\ \mathrm{l}_{21} & \mathrm{l}_{22} & 0 \\ \mathrm{l}_{31} & \mathrm{l}_{32} & \mathrm{l}_{33}\end{array}\right]\left[\begin{array}{ccc}\mathrm{u}_{11} & \mathrm{u}_{12} & \mathrm{u}_{13} \\ \mathrm{u}_{21} & \mathrm{u}_{22} & 0 \\ \mathrm{u}_{31} & 0 & 0\end{array}\right]=\left[\begin{array}{lll}\mathrm{a}_{11} & \mathrm{a}_{12} & \mathrm{a}_{13} \\ \mathrm{a}_{21} & \mathrm{a}_{22} & \mathrm{a}_{23} \\ \mathrm{a}_{31} & \mathrm{a}_{32} & \mathrm{a}_{33}\end{array}\right]$

it can easily verify that $\mathrm{a}_{11}=\mathrm{m}_{11} \mathrm{n}_{11}$. If $\mathrm{k}_{11}=0$, either $\mathrm{m}_{11}=0$ or $\mathrm{n}_{11}=0$ which shows that either matrix $m=0$ or matrix $n=0$. The matrix $m$ is equivalent to $\mathrm{L}$ and $\mathrm{n}$ is equivalent to $\mathrm{U}$.

\subsection{Singular value Decomposition (SVD)}

A matrix A of size mxn can be decomposed as [10]

$$
\mathrm{A}=\mathrm{UDV}^{\mathrm{T}}
$$

$\mathrm{U}$ is a column orthogonal matrix of size mxn and its columns are eigen vectors of $\mathrm{AA}^{\mathrm{T}}$

$$
\text { i.e, } A A^{\mathrm{T}}=U \mathrm{UDV}^{\mathrm{T}} \mathrm{VDU}^{\mathrm{T}}=\mathrm{UD}^{2} \mathrm{U}^{\mathrm{T}}
$$

$\mathrm{V}$ is a orthogonal matrix of size nxn and its columns are eigen vectors of $A^{\mathrm{T}} \mathrm{A}$

$$
\text { i.e, } A^{\mathrm{T}} \mathrm{A}=V D U^{\mathrm{T}} U D V^{\mathrm{T}}=V^{2} \mathrm{~V}^{\mathrm{T}}
$$

$\mathrm{D}$ is a diagonal matrix of size nxn called singular values.

$$
\begin{aligned}
& \text { If } \mathrm{U}=\left(\mathrm{u}_{1} \mathrm{u}_{2} \ldots \ldots \mathrm{u}_{\mathrm{n}}\right) \text { and } \mathrm{V}=\left(\mathrm{v}_{1} \mathrm{v}_{2} \ldots \ldots \mathrm{v}_{\mathrm{n}}\right) \text { then } \\
& \qquad A=\sum_{i=1}^{n} \sigma_{i} u_{i} v_{i}^{T}
\end{aligned}
$$

\subsection{Kronecker Product}

The symbol $\otimes$ can be used to represent the kronecker product. Let $\mathrm{X}$ and $\mathrm{Y}$ are two matrices then the kronecker product of two matrices can be represented as $\mathrm{X} \otimes \mathrm{Y}$. Let the matrix $\mathrm{X}$ of size $\mathrm{p} \mathrm{x} \mathrm{q}$ and the matrix $\mathrm{Y}$ of size $\mathrm{r} \mathrm{x}$ s then the resulting matrix of size pr $\mathrm{x}$ qs

\subsection{Katric Rao Product}

$$
\mathrm{X} \otimes \mathrm{Y}=\left[\begin{array}{c}
\mathrm{x}_{11} \mathrm{Y} \ldots . \mathrm{x}_{1 \mathrm{q}} \mathrm{Y} \\
\vdots \\
\vdots \\
\mathrm{x}_{\mathrm{p} 1} \mathrm{~B} \ldots \mathrm{x}_{\mathrm{pq}} \mathrm{Y}
\end{array}\right]
$$

The Khatri-Rao product is a column-wise Kronecker product. Originally introduced by Khatri and Rao (1968) [7].

Given matrices $A \in R^{I \times K}$ and $B \in R^{J \times K}$ their KhatriRao product is denoted by $\mathrm{A} \otimes \mathrm{B}$. The result is a matrix of size $(\mathrm{IJ}) \times \mathrm{K}(\mathrm{IJ}) \times \mathrm{K}$ and defined by [8]

$$
\mathrm{A} \otimes \mathrm{B}=\left[\mathrm{a}_{1} \otimes \mathrm{b}_{1} \mathrm{a}_{2} \otimes \mathrm{b}_{2} \ldots \mathrm{a}_{\mathrm{k}} \otimes \mathrm{b}_{\mathrm{k}}\right]
$$

\section{DECISION STRATEGY - MEAN SQUARE ERROR (MSE)}

In the proposed model, the MSE is used as for verification with the support of feature and selection process at the training and testing levels.

\section{Mean Square Error:}

The Mean Square Error (MSE) of an estimator $\widehat{X}$ of a parameter $\mathrm{X}$ is the function of $\mathrm{X}$ defined by $\mathrm{E}(\widehat{\mathrm{X}}-\mathrm{X})^{2}$ and it is denoted as $\mathrm{MSE}_{\widehat{\mathrm{X}}}$.

$$
\operatorname{MSE} \widehat{(X)}=E\left[(\widehat{X}-X)^{2}\right]
$$

The Mean Square Error is equal to the sum of the variance and the squared bias of the estimator or of the predictions. In the case of the MSE of an estimator,

$$
\operatorname{MSE}(\widehat{X})=\operatorname{Var}(\widehat{X})+(\operatorname{Bias}(\widehat{X}, \mathrm{X}))^{2}
$$

\section{EXPERIMENT ANALYSIS}

By considering benchmark Yale and AT\&T, FERET data sets [12,13], several experiments have been conducted. The MSE is considered as decision process. The experimental results on the chosen data is listed in the Table 1 with different key sizes such as $8 \times 8,16 \times 16,24 \times 24,32 \times 32 \ldots . .64 \times 64$ on similar and dissimilar patterns.

This model uses PCA for normalization and LU factorization for feature selection and extraction [5]. Later trained images can be projected by using SVD and weights can be calculated. These weights can be encoded with Katri Rao product [7]. Similarly testing image weights can be calculated and these weights can be compared with training image weights. Mean Square Error (MSE) was used for comparison. In this, we took, threshold value $\mathrm{d}=0.12$ and the results obtained were implemented on Core i 7 th generation processor with 16 GB of RAM. From the observations of MSE and False Acceptance Rate (FAR) and False Non Acceptance Rate (FNAR) have been measured for the chosen key sizes of the patterns. The algorithm works better for all key sizes. 
Available online at www.ijrat.org

\begin{tabular}{|c|l|l|l|l|}
\hline \multirow{2}{*}{ S No } & \multirow{2}{*}{ Key Size } & \multicolumn{3}{|l|}{ Mean Square Error (MSE) } \\
\cline { 3 - 5 } & & Completely Similar & $\begin{array}{l}\text { Similar with different } \\
\text { poses }\end{array}$ & Dissimilar \\
\hline 1 & $8 \times 8$ & 0.000000 & 0.040750 & 0.182083 \\
\hline 2 & $16 \times 16$ & 0.000000 & 0.106621 & 0.136280 \\
\hline 3 & $24 \times 24$ & 0.000000 & 0.108528 & 0.141979 \\
\hline 4 & $32 \times 32$ & 0.000000 & 0.093009 & 0.165426 \\
\hline 5 & $40 \times 40$ & 0.000000 & 0.114700 & 0.152512 \\
\hline 6 & $48 \times 48$ & 0.000000 & 0.086422 & 0.195976 \\
\hline 7 & $56 \times 56$ & 0.000000 & 0.076474 & 0.187708 \\
\hline 8 & $64 \times 64$ & 0.000000 & 0.091097 & 0.205079 \\
\hline
\end{tabular}

Table 1: Results

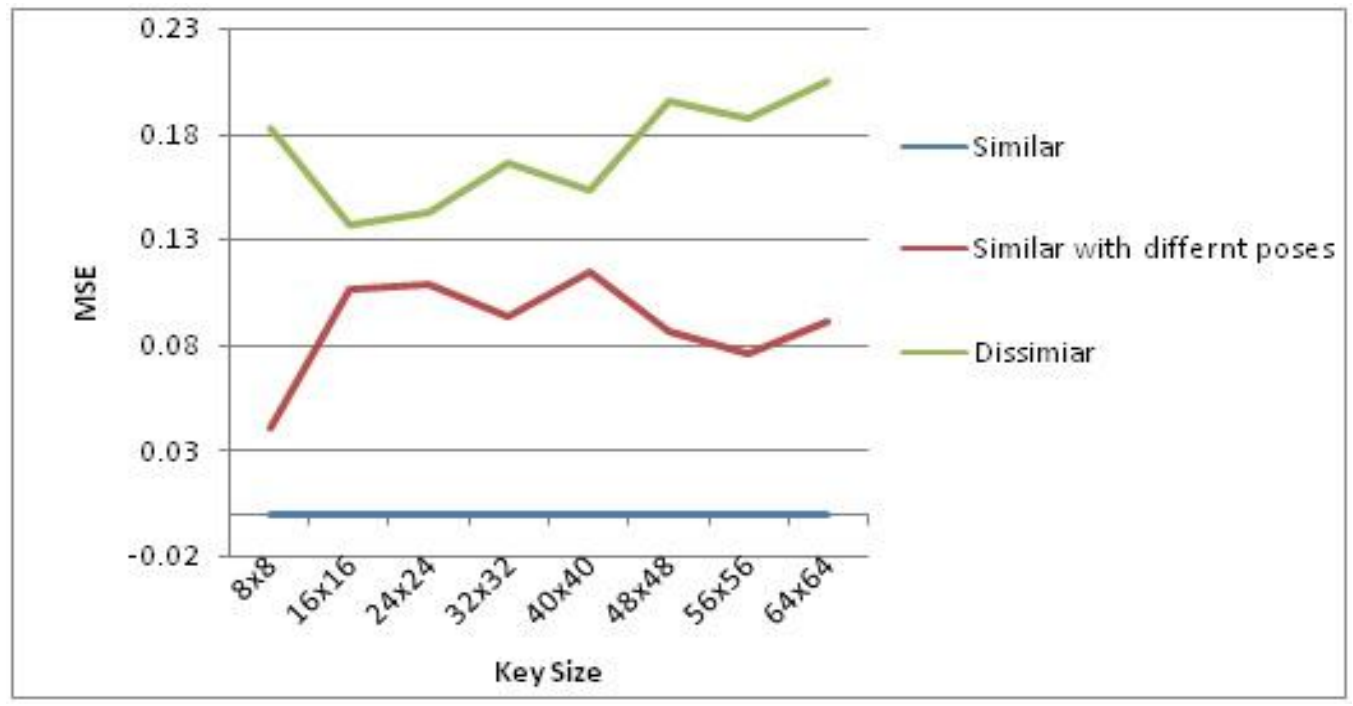

Fig.2. Graphical representation of results.

\section{Acknowledgements}

I gratefully acknowledge the funding agency, the University Grant Commission (UGC) of the Government of India, for providing financial support through UGC-SERO (MRP-6521/16).

\section{REFERENCES}

[1] Multimodal Biometric for Person Authentication by Fusion, C. Nandini and C. N. Ravi Kumar, TECHNIA-International Journal of Computing Science and Communication Technologies, VOL. 2, NO. 1, July 2009. (ISSN 0974-3375)

[2] Biometric Identification through Hand Geometry Measurements, Raul Sanchez-Reillo, Carmen Sanchez-Avila, Ana Gonzalez-Marcos "IEEE Transaction on Pattern Analysis and Machine Intelligence:, Vol.22, No.10, October 2000.

[3] Wu, X; Wang, K; Zhang, D (2004). A novel approach of palm-line extraction; in: Proceeding of the Third International Conference on Image and Graphics.
Rasmus Elsborg Madsen, Lars Kai Hansen and Ole Winther, "Singular Value Decomposition and Principle Component Analysis", February 2004

[5] Y Suresh, "Feature Selection in Multimodal Authentication using LU Factorization with CSEAM Model", International Journal onComputational Sciences \& Applications (IJCSA) Vol.5, No.1, February 2015.

[6] M. Andy Kass and Yaoguo Li, "Use of principal component analysis in the de-noising and signal separation of transient electromagnetic data" URL: https://pdfs.semanticscholar.org/71c0/44b3dafba2d 12826cbed9fe473a161c42c49.pdf

[7]https://stat.ethz.ch/R-manual/R-devel/library/ Matrix/html/KhatriRao.html

[8]https://math.stackexchange.com/questions/ 1916069/ khatri-rao-product-example

[9]http://www.advancedsourcecode.com/fingerprintdat abase.asp

[10] Izquierdo-Fuento, A.; del Val, L.; Jimenez M.I.; Villakorta, J.J. "Performance Evaluation of a 


\section{E-ISSN: 2321-9637}

\section{Available online at $w w w . i j r a t . o r g$}

Biometric System Based on Acoustic Images. Sensors, 119499-9519, 2011.

[11] Y Suresh, "Pattern Recognition Using Context Dependent Memory Model (CDMM) In Multimodal Authentication System", International Journal in Foundations of Computer Science \& Technology (IJFCST), Vol.5, No.1, January 2015.

[12] http://www.advancedsourcecode.com/fingerprint database.asp

[13] http://www.face-rec.org/databases/

[14] Siddhartha Bhattacharya, Paramartha Dutta, Sourav De. Goran Klepac, "Hybrid Soft Computing for Image Segmentation", Springer Publication, 2016.

[15] Ning Chen, Mingyu Li and Haidong Xiao, "Twolayer similarity fusion model for cover song identification", EURASIP Journal on Audio, Speech and Music Processing(2017:12), DOI 10.1186/s13636-017-0108-2.

[16] Image Segmentation using Varieties of Auto Encoders in Tensorflow, https://towardsdatascience.com/imagesegmentation-using-varieties-of-auto-encoders-intensorflow-manual-back-prop-with-tf2e688f2a98f7.

[17] Yiqing Zhao, Nooshin J. Fesharaki, Hongfang Liu and Jake Luo, "Using data-driven sublanguage pattern mining to induce knowledge models: application in medical image reports knowledge representation", BMC Medical Informatics and Decision Making (2018) 18:61 https://doi.org/10.1186/s12911-018-0645- .

[18] Irhum Shafkat, "Intuitively Understanding Convolutions for Deep Learning", https://towardsdatascience.com/intuitivelyunderstanding-convolutions-for-deep-learning1f6f 42 faee 1 